\title{
MODELLING OF SOLAR WIND, CME INITIATION AND CME PROPAGATION
}

\author{
B. VAN DER HOLST, S. POEDTS*, E. CHANÉ, C. JACOBS, G. DUBEY and D. KIMPE \\ Centrum Voor Plasma-Astrofysica, K.U.Leuven, Celestijnenlaan 200B, B-3001 Leuven, Belgium \\ (*Author for correspondence: E-mail: Stefaan.Poedts@wis.kuleuven.be)
}

(Received 31 July 2005; Accepted in final form 9 January 2006)

\begin{abstract}
Simulations of coronal mass ejections (CMEs) evolving in the interplanetary (IP) space from the Sun up to $1 \mathrm{AU}$ are performed in the framework of ideal magnetohydrodynamics (MHD) by the means of a finite-volume, explicit solver. The aim is to quantify the effect of the background solar wind and of the CME initiation parameters, such as the initial magnetic polarity, on the evolution and on the geo-effectiveness of CMEs. First, three different solar wind models are reconstructed using the same numerical grid and the same numerical scheme. Then, different CME initiation models are considered: Magnetic foot point shearing and magnetic flux emergence. For the fast CME evolution studies, a very simple CME model is considered: A high-density and high-pressure magnetized plasma blob is superposed on a background steady state solar wind model with an initial velocity and launch direction. The simulations show that the initial magnetic polarity substantially affects the IP evolution of the CMEs influencing the propagation velocity, the shape, the trajectory (and thus, the geo-effectiveness).
\end{abstract}

Keywords: computational MHD, solar wind CME initiation, CME propagation, space weather

\section{Introduction}

During October and November 2003, a series of solar eruptions occurred and gave rise to some of the largest geomagnetic storms of the solar cycle. During this period, $59 \%$ of the reporting spacecrafts experienced some effects from the solar activity (Gopalswamy et al., 2005). It is well known that geomagnetic storms can perturb and interrupt electric power equipment, cause radio blackouts and navigation system failures, and damage satellite systems. Surprisingly, it seems that sperm whale strandings are also related to solar activity (Vanselow and Ricklefs, 2005). In order to improve the geomagnetic storm predictions, studies on CME evolution are really important.

Gombosi et al. (2000) numerically simulated a coronal mass ejection (CME) propagating from the Sun to the Earth interacting with the magnetosphereionosphere system. Manchester et al. (2004a) then performed the same kind of simulation but with a Gibson and Low CME model in a first step up to $32 R_{\odot}$, and later up to $1 \mathrm{AU}$ (Manchester et al., 2004b). More recently, Lugaz et al. (2005) used the data from a numerical simulation to reproduce line of sight images or even to give a stereographic view of CMEs as it would be observed by the Solar Terrestrial Relations Observatory (STEREO) project.

Space Science Reviews (2005) 121: 91-104

DOI: $10.1007 / \mathrm{s} 11214-006-6541-7$

(C) Springer 2006 
In fact, a lot of people are doing numerical simulations of CMEs but everyone uses his/her own background wind model and favorite CME model so that it becomes difficult, if not impossible, to compare the results of different authors (see, e.g., Lionello et al. (2003), Roussev et al. (2003a), Lee et al. (2004), Odstrcil et al. (2004), Odstrcil et al. (2005), Amari et al. (2005), Lionello et al. (2005), Lugaz et al. (2005)). Jacobs et al. (2005) made a study on the effect of the applied background wind model on the IP CME evolution. Chané et al. (2005) extended this study and showed the importance of the magnetic polarity of the initial CME flux rope on the evolution of the CME. Unfortunately, in these simulations the computational domain stopped at $30 R_{\odot}$ due to lack of CPU power. In the present paper, however, the computational domain is extended up to $1 \mathrm{AU}$. This enables us to produce in situ data close to the Earth in order to fully investigate the influence of the initial magnetic polarity on the geo-effectiveness of the CMEs.

The applied wind models are presented in Section 2. In Section 3, the CME initiation is explained and in Section 4 the ICME simulations are presented. The important characteristics are explained and discussed for the inverse and for the normal CMEs.

\section{The Solar Wind Models}

We reconstructed three models for the solar wind that are often used in the literature. All three models are axi-symmetric (2.5D, i.e. two coordinate $r$ and $\theta$ and keeping all three vector components for the velocity and magnetic field) and were reconstructed with the same numerical code VAC (Versatile Advection Code; Tóth (1996)), with the same numerical technique, namely the Lax-Friedrichs scheme, on the same grid, and with the same boundary conditions such that the only difference between the winds lies in the physics included in the equations. More details can be found in (Jacobs et al., 2005).

The simulation domain extends to $30 R_{\odot}$ for the CME initiation studies and to $1 \mathrm{AU}$ for the CME evolution studies and are both $2.5 \mathrm{D}$ also.

The first model is a classical polytropic model with polytropic relation: $p=$ $\rho^{\gamma} / \gamma$, where $\gamma=1.05$. This wind model is fairly popular in the literature in spite of its remarkable shortcomings. It has, e.g., a too small ratio of polar versus the equatorial wind velocity. Wind model 2 is a reconstruction of the MHD wind model, used and described by Manchester et al. (2004a), where the full set of ideal MHD equations is solved. It is obtained by means of an extra heating source term in the energy equation which, in normalized form, is given by

$$
Q=\rho q_{0} \exp ^{-\frac{(r-1)^{2}}{\sigma^{2}}}\left(T_{0}-\gamma \frac{p}{\rho}\right),
$$

with the profiles of $T_{0}$ and $\sigma$ having both a $\theta$ - and $r$-dependence. This heating source term yields a much better wind model than the polytropic wind, with the 
right velocity ratio. The third model is again a polytropic model but now including an additional pressure gradient due to Alfvén waves. The extra Alfvén wave pressure, $p_{w}$, in the momentum equation is determined by

$$
\frac{\partial \epsilon_{ \pm}}{\partial t}+\nabla \cdot\left[\left(\mathbf{v} \pm \mathbf{v}_{\mathrm{A}}\right) \epsilon_{ \pm}\right]=-\frac{1}{2} \epsilon_{ \pm} \nabla \cdot \mathbf{v}
$$

where $\epsilon_{ \pm}$is the average Alfvén wave density for the parallel and antiparallel propagating waves and $\mathbf{v}_{A}$ is the Alfvén velocity. The extra Alfvén wave pressure is given by

$$
p_{\mathrm{w}}=\frac{\epsilon_{+}+\epsilon_{-}}{2} .
$$

The three winds are illustrated in the upper row of Figure 1 showing the density (green scale), the magnetic field lines, the velocity vectors. The total rate of change of angular momentum of the Sun due to the wind is equal to minus the angular momentum flux at any given radius. For $2.5 \mathrm{D}$ axi-symmetric winds this is

$$
\frac{d J_{\mathrm{z}}}{d t}=-2 \pi \int_{0}^{\pi} r^{3} \sin ^{2} \theta\left(\rho v_{\mathrm{r}} v_{\phi}-B_{\mathrm{r}} B_{\phi}\right) d \theta .
$$

The first term between brackets represents the angular momentum flux due to advection, the second term is due to the magnetic tension. In Figure 1, the angular momentum flux as well as the advection and magnetic tension part is shown, indicating that the angular momentum flux is conserved by the numerical code. The second wind model was extended to $1 \mathrm{AU}$ for the CME evolution studies. The resulting $\theta$-profiles of the solar wind density and radial velocity at $1 \mathrm{AU}$ are shown in Figure 2.

\section{CME Initiation Models}

In this paragraph, the sub-problem of the CME initiation is handled. Two popular models for CME initiation are foot point shearing and magnetic flux emergence (or cancellation). Both these initiation models are superposed on the different wind models to examine effect of the background wind on the CME initiation. The effect of the initiation parameters (e.g. shear velocity, flux emergence rate, etc.) on the development of the CMEs is also investigated.

\subsection{Foot Point SHEARING}

In order to trigger an instability of the magnetic structure (CME), the foot points of the magnetic field are sheared by adding an extra longitudinal velocity at the 

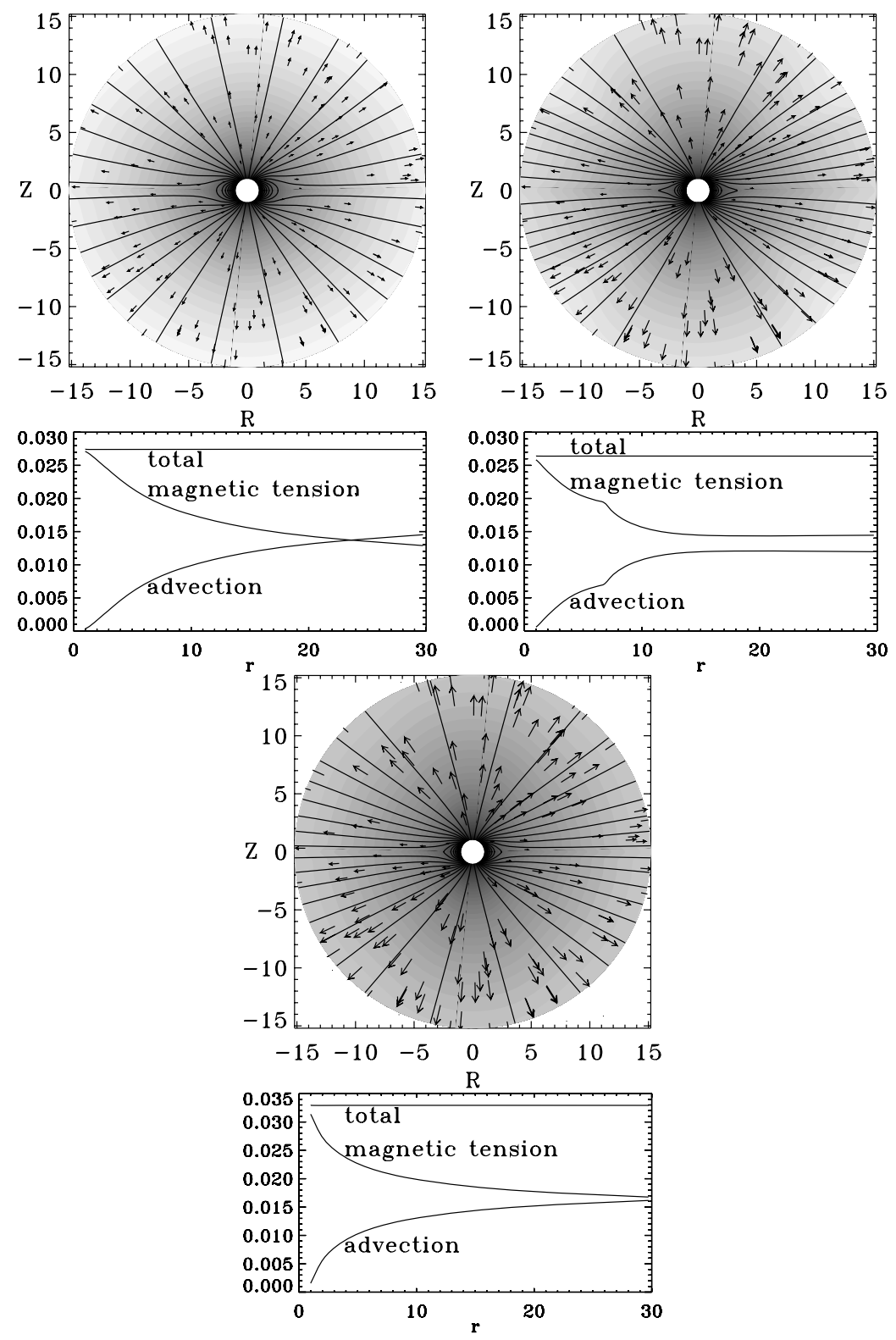

Figure 1. The three steady wind solutions and the corresponding total angular momentum fluxes. Upper left: The (standard) polytropic model with $\gamma=1.05$; upper right: MHD wind model with energy source term; bottom: Polytropic MHD wind model with Alfvén wave acceleration term. The green scale represents the density (log-scale), the black lines are magnetic field lines, arrows denote the radial velocity. 


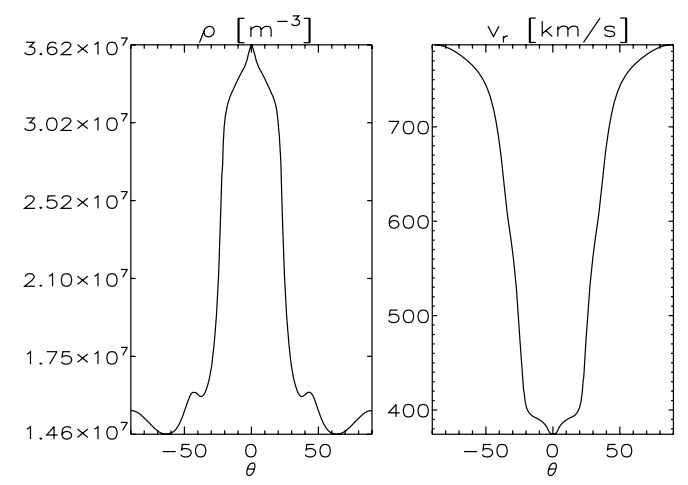

Figure 2. The $\theta$-profiles of the solar wind density and radial velocity at $1 \mathrm{AU}$.

solar surface in accordance with Mikic and Linker (1994). The amount of added longitudinal velocity is given by

$$
v_{\varphi}^{0}=v_{0}(t) \Theta \exp \left[\frac{1-\Theta^{4}}{4}\right],
$$

where $\Theta=(\theta-90) / \Delta \theta_{m}, \theta$ is the co-latitude in degrees, and $v_{0}(t)$ a function that specifies the time profile. The simulation stops when a time $t_{\max }=180 \mathrm{~h}$ is reached. The shearing reaches its maximum value $\Delta \theta_{\mathrm{m}}$ degrees above and below the equator. In the simulations performed we took $\Delta \theta_{\mathrm{m}}$ equal to $20^{\circ}$ and the maximum shear velocity varied between values of 3,6 , or $9 \mathrm{~km} / \mathrm{s}$. The three different models for the solar wind, described above, were used as background wind.

In Figure 3, the relative density is shown for successive snapshots in the evolution of the CME formation. The background wind is the standard polytropic model and the shear velocity measures $6 \mathrm{~km} / \mathrm{s}$. By shearing the foot points, the helmet streamer starts swelling until it becomes unstable and a flux rope is formed. Although the ideal MHD equations are solved, numerical dissipation cannot be excluded and allows the formation of a flux rope by reconnection processes. The relative density is given by $\left(\rho_{\text {tot }}-\rho_{\mathrm{w}}\right) / \rho_{\mathrm{w}}$, with $\rho_{\text {tot }}$ the total density and $\rho_{\mathrm{w}}$ the density of the stationary background wind.

The model for the background wind affects the time of formation of the flux rope. This can be seen in Figure 4 where a plot is shown of the evolution of the total magnetic energy in time and the maximum in magnetic energy is reached right before the formation of the flux rope. The amount of magnetic energy is expressed with respect to the total amount of magnetic energy in the stationary background wind. Because the shearing is a continuous process, several flux ropes will be formed.

The faster the shearing, the earlier the flux rope is formed. Also the time-interval between the succeeding flux ropes scales with the shearing velocity. The shear velocity also determines if a flux rope will be formed or not. Shearing at a too low velocity will not lead to the formation of a flux rope, independent of the shearing 

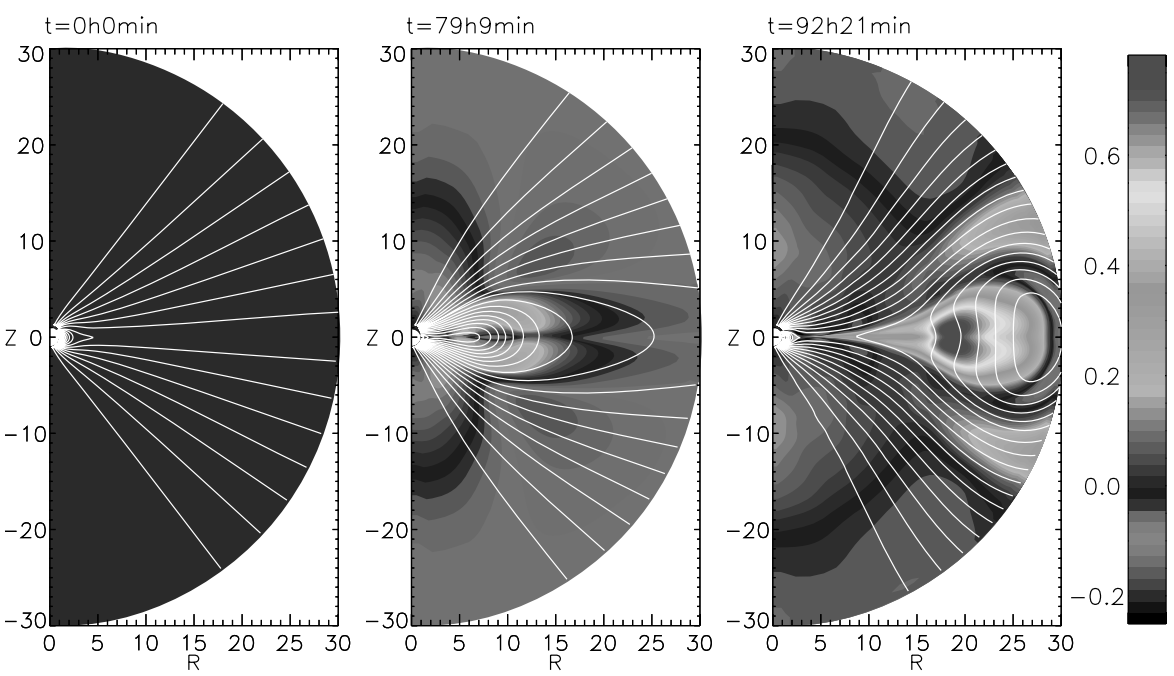

Figure 3. Evolution of the relative density and formation of a flux rope by foot point shearing. Wind model $1, v_{\phi}^{\max }=6 \mathrm{~km} / \mathrm{s}$.

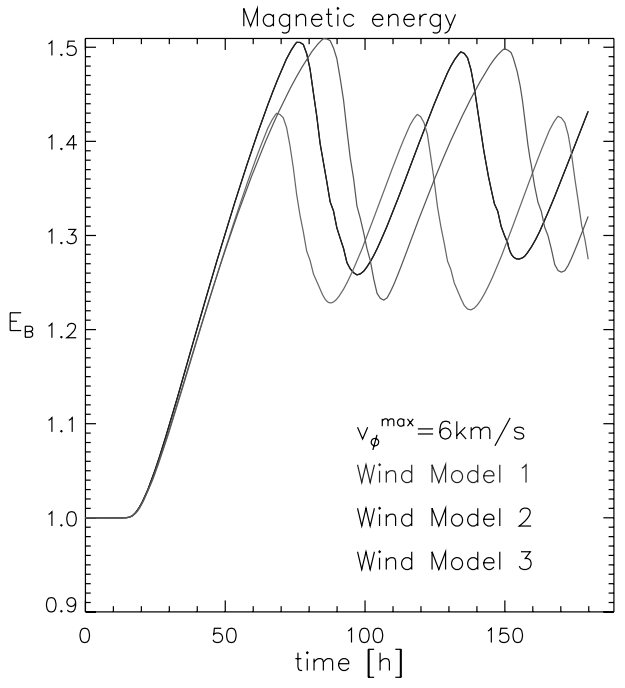

Figure 4. The evolution of the magnetic energy in time, for maximum shear velocity of $6 \mathrm{~km} / \mathrm{s}$.

time interval (see Jacobs et al., 2006). This is the case for shearing at $3 \mathrm{~km} / \mathrm{s}$ in wind models 2 and 3. The amount of extra longitudinal velocity added also influences the velocity at which the flux rope moves through interplanetary space. This can be seen in the left part of Figure 5 where the velocity of the center of the flux rope is plotted against position of the flux rope center. This curve is obtained by taking the derivative with respect to time of the height-time curves of the center of the 


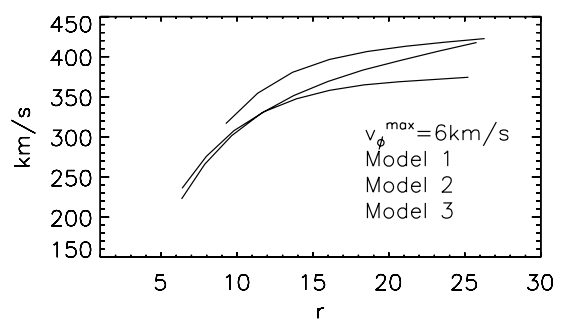

Figure 5. Velocity of the flux rope in the different wind models for $v_{\phi}^{\max }=6 \mathrm{~km} / \mathrm{s}$.

flux rope. From this we can see that the faster the field lines are sheared, the faster the flux rope is moving. Also the background wind has an influence on the velocity of the flux rope. This can be seen in Figure 5. For the given shearing velocities it turns out impossible to create fast CMEs with this initiation mechanism. For more details see Jacobs et al. (2006).

\subsection{Magnetic Flux Emergence}

CMEs can also be triggered by the emergence of additional magnetic flux of the same or the opposite polarity as the overlying magnetic field. Dubey et al. (2005) considered a flux rope in a dipole magnetic field with a density that is 200 times higher that at the solar surface. The flux rope is initially kept stable by means of a line current (cf. Chen and Shibata (2000)). We then allow magnetic flux of the opposite polarity to emerge from the solar surface at a certain rate. This additional magnetic flux breaks the force balance and causes the CME to be launched (see Figure 6). The evolution parameters, such as the velocity and the acceleration, depend on the flux emergence rate and on the total amount of flux that is emerged and we obtain velocities in the order of $350-400 \mathrm{~km} / \mathrm{s}$, which is precisely in the range of what is observed for the majority of the CMEs. However, Dubey et al. (2005) were not able to create fast CMEs (with velocities $>1000 \mathrm{~km} / \mathrm{s}$ ) in this way. The next steps will, therefore, be to extend the parameter study and to replace the background dipole magnetic field by a solar wind model.

\subsection{CME Models With Initial Density and Velocity Specification}

In order to study the propagation of fast CMEs and CME shocks in the interplanetary space from the solar corona up to $1 \mathrm{AU}$, a very simple model is used to generate the CMEs in the present paper. This model is far to be a state-of-the-art CME initiation model with very complex features, but it gives us an easy way to study the importance of the initial magnetic polarity of the CME. A high-density and highpressure magnetized plasma blob is superposed on the background steady-state solar wind model. The CMEs are launched at a certain velocity $v_{\text {cme }}$, in a prescribed radial 

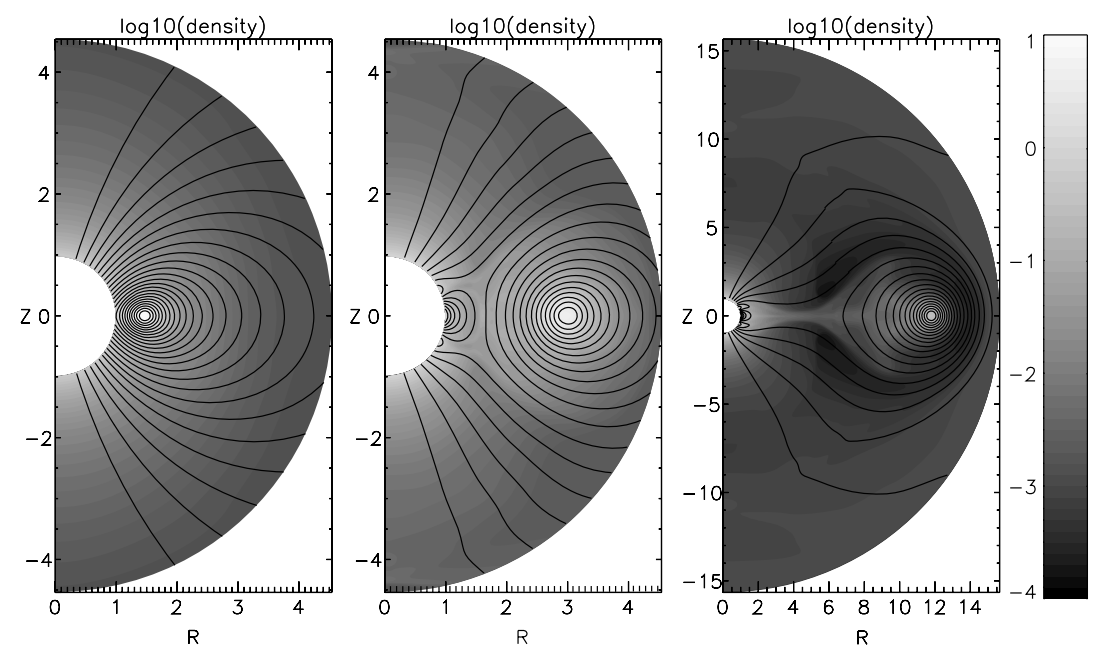

Figure 6. Evolution of the plasma density $\log _{10} \rho$ (grey scale) and magnetic field lines at $39 \mathrm{~h} 17 \mathrm{~min}$ (left), $40 \mathrm{~h} 31 \mathrm{~min}$ (middle), and at $46 \mathrm{~h} 39 \mathrm{~min}$ (right, with a different scale on the abscis) for the case with flux emergence that starts at $39 \mathrm{~h} 17 \mathrm{~min}$ and stops at $39 \mathrm{~h} 27 \mathrm{~min}$.

direction $\theta_{\text {cme. }}$. The velocity and density profile in the initial disturbance are both of the form:

$$
y=\frac{y_{\mathrm{cme}}}{2}\left(1-\cos \pi \frac{d_{\mathrm{cme}}-d}{d_{\mathrm{cme}}}\right),
$$

where $y$ indicates the density $\rho$ or the radial velocity $v_{\mathrm{r}}, y_{\mathrm{cme}}$ is the maximum density or radial velocity in the plasma bubble, $d_{\text {cme }}$ is the radius of the bubble and $d$ the distance to the center of the bubble. The CMEs are further characterized by a given density, magnetic field strength and magnetic polarity. The initial CME magnetic field and the background wind magnetic field can have the same or the opposite polarity. We call it respectively an inverse and a normal CME, according to the nomenclature of Low and Zhang (2002) (see Chané et al. (2005) for more details about the CME initiation ).

For the CMEs discussed in the present paper, the radius of the initial plasma blob, $d_{\text {cme }}$, is $0.29 R_{\odot}$ and its center $d$ is located at $1.5 R_{\odot}$. In the center of the initial perturbation, the velocity is about $1000 \mathrm{~km} / \mathrm{s}$ and the density is five times higher than the density on the surface of the Sun. In the initial plasma blob, the maximal magnetic field strength is $\sim 3.44 \mathrm{G}(\sim 0.344 \mathrm{mT})$. In all the numerical simulations discussed in this paper, the CMEs have exactly the same initial density, pressure, velocity, and magnetic strength. The only difference will be the initial magnetic polarity: inverse or normal. In the following section, the influence of the initial magnetic polarity on the time of arrival, on the evolution path, and on the geo-effectiveness of the ICMEs will be investigated. 


\section{CME Evolution}

It turns out that the polarity of the flux rope magnetic field has a great influence on the evolution of the CME. The polarity influences the mass distribution inside the CME, the spread angle, the evolution path, and also the velocity of the shock front. When launching a magnetized CME outside the equatorial plane, the magnetic forces push the CME towards or away from the equator, depending if the polarity of the flux rope is inverse or normal. Also it is seen that the normal magnetized CME moves slightly faster than the magnetized inverse (for a physical explanation of this, see Chané et al., 2005b). This has consequences for predicting the time of arrival and geo-effectiveness of a CME.

In spite of the rather simple and naive CME model used in these simulations, they yield some very good and realistic results. First of all we were able to reproduce the magnetic topology predicted by Low and Zhang (2002). Behind the CME, in the equatorial plane, reconnection processes occur, which lead to a gradually rebuilding of the helmet streamer and yield back flows along the helmet streamer, very much as seen in observations. Another interesting fact is that we can extend these CME simulations to $1 \mathrm{AU}$ and that we can reproduce some of the cases in the ACE database, where the strength jump in magnetic field, the profile for the velocity, and the time of arrival are matching surprisingly well.

\subsection{Characteristics of The CMEs}

In this subsection, we will try to point out the characteristic shape (density repartition and magnetic field configuration) of the normal and the inverse CMEs. Figure 7 shows an inverse and a normal CME launched in the equatorial plane, travelling in the IP space. Clearly the shock front travels slower close to the equator resulting in a strange shape transformation of the CMEs. This is due to the wind model we are using that presents a higher density and a lower velocity on the equator (see Figure 2). After $9 \mathrm{~h} 36 \mathrm{~min}$ the magnetic cloud still has an almost circular shape. But after $55 \mathrm{~h} 11 \mathrm{~min}$ the magnetic cloud is so compressed that it is hard to recognize the Low and Zhang configuration and the CME evolution is thus far from self-similar. The density repartition of an inverse and a normal CME is, in fact, quite similar at $1 \mathrm{AU}$. They are both characterized by a shock front evolving in front of the magnetic cloud, which contain the main part of the density. The magnetic field lines are not that different for the two CMEs although the magnetic cloud seems to be more deformed (less circular) for the normal CME.

Figure 8 mimics the data obtained by a spacecraft at $1 \mathrm{AU}$ when these CMEs would pass it. One can see that the normal CME shock front arrives at 1 AU only $4 \mathrm{~h}$ before the inverse shock front. The inverse CME displays a strong southward magnetic field, which may lead to a magnetic storm and the normal CME displays a strong northward magnetic field which normally does not cause a magnetic storm. 


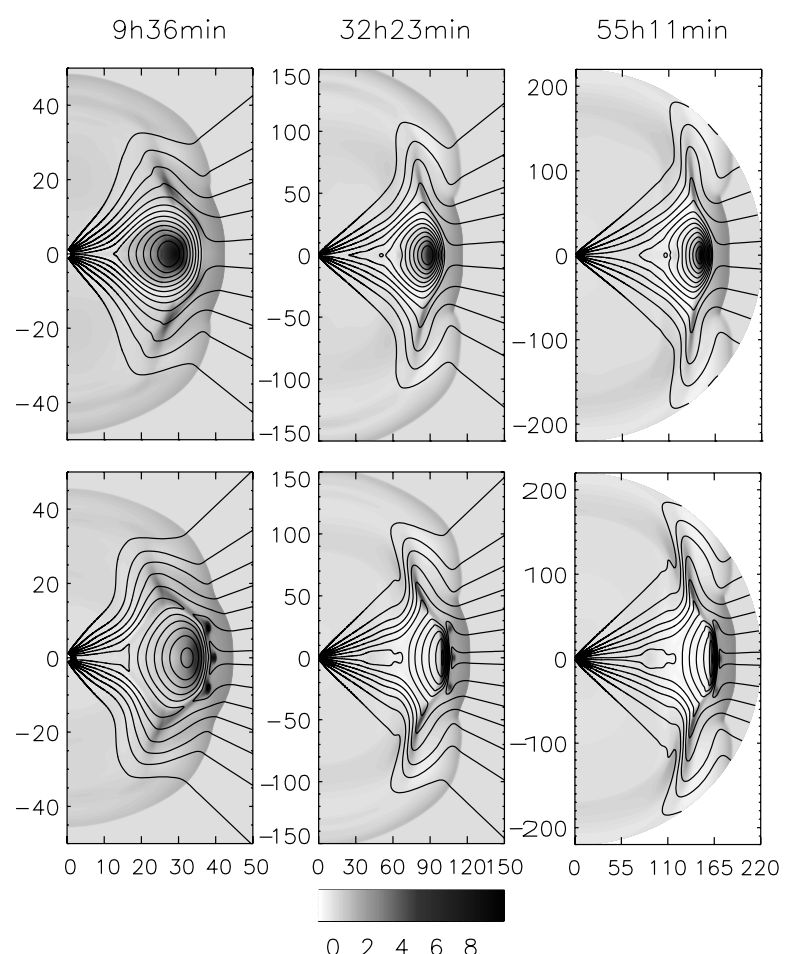

Figure 7. Contour plots of the relative density distribution for a CME launched in the equatorial plane $\left(\theta_{\text {ini }}=0^{\circ}\right)$. Upper row: inverse initial magnetic polarity; lower row: normal initial magnetic polarity. left column: after $9 \mathrm{~h} 36 \mathrm{~min}$; central column: after $32 \mathrm{~h} 23 \mathrm{~min}$; right column: after $55 \mathrm{~h} 11 \mathrm{~min}$. The black lines represent the projected magnetic field lines. The relative density is defined as follows: $\bar{\rho}=\left(\rho-\rho_{\text {wind }}\right) / \rho_{\text {wind }}$.

The velocity of the normal CME is a little bit higher and so this CME reaches the Earth a few hours earlier than the inverse CME.

\subsection{Evolution Path}

When a CME travels in the IP space, it is really important to know its trajectory, i.e., whether its evolution path is a straight line or whether it deviates from this line and, if so, how much. In some cases, this information can be crucial to predict if a CME will hit or miss the Earth. Chane et al. (2005) have studied the evolution path of magnetized CMEs up to $30 R_{\odot}$ and have shown that the initial magnetic polarity of the CME flux rope affects its evolution path substantially: normal CMEs are deviated towards the pole while inverse CMEs are deviated towards the equatorial plane, which means that the probability to hit the Earth should be higher for inverse CMEs. We will show that these conclusions remain partially true up to 1 AU. Figure 9 shows the results of several simulations for 

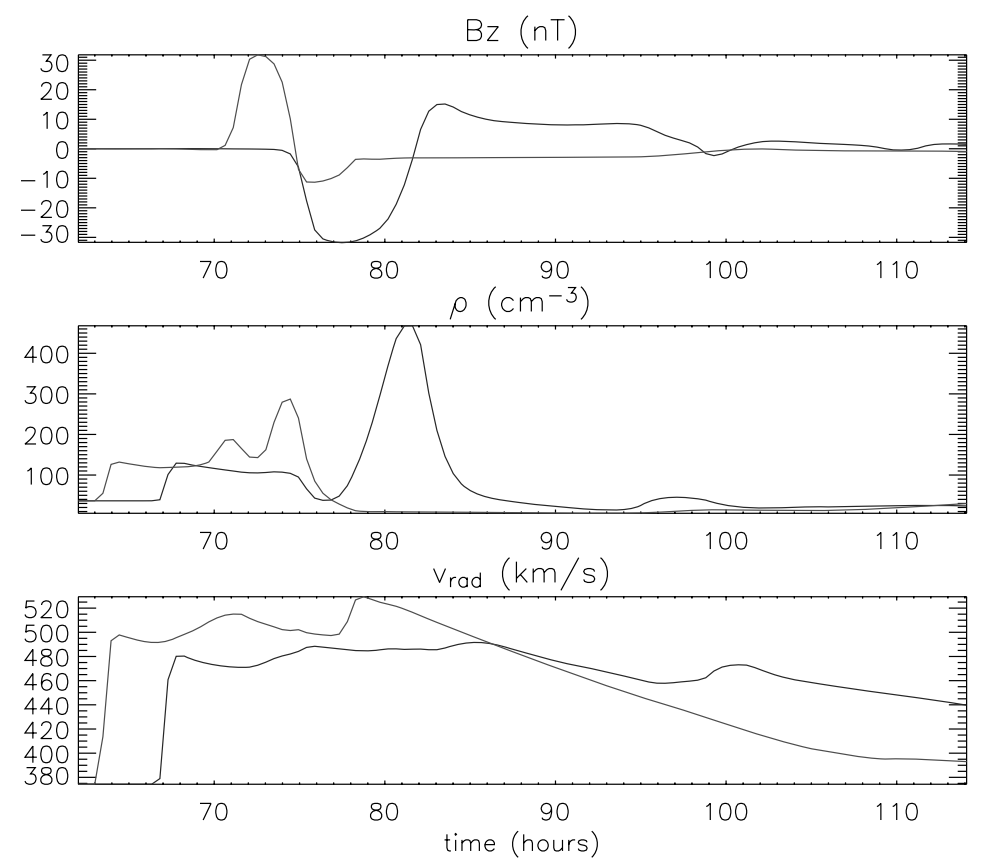

Figure 8. Evolution of the magnetic field, density, and velocity in time in the equatorial plane $\left(\theta=0^{\circ}\right)$ at a position of $215 R_{\odot}$ for a $\mathrm{CME}$ launched in the equatorial plane $\left(\theta_{\mathrm{cme}}=0^{\circ}\right)$; red lines: normal CME; blue lines: inverse CME.

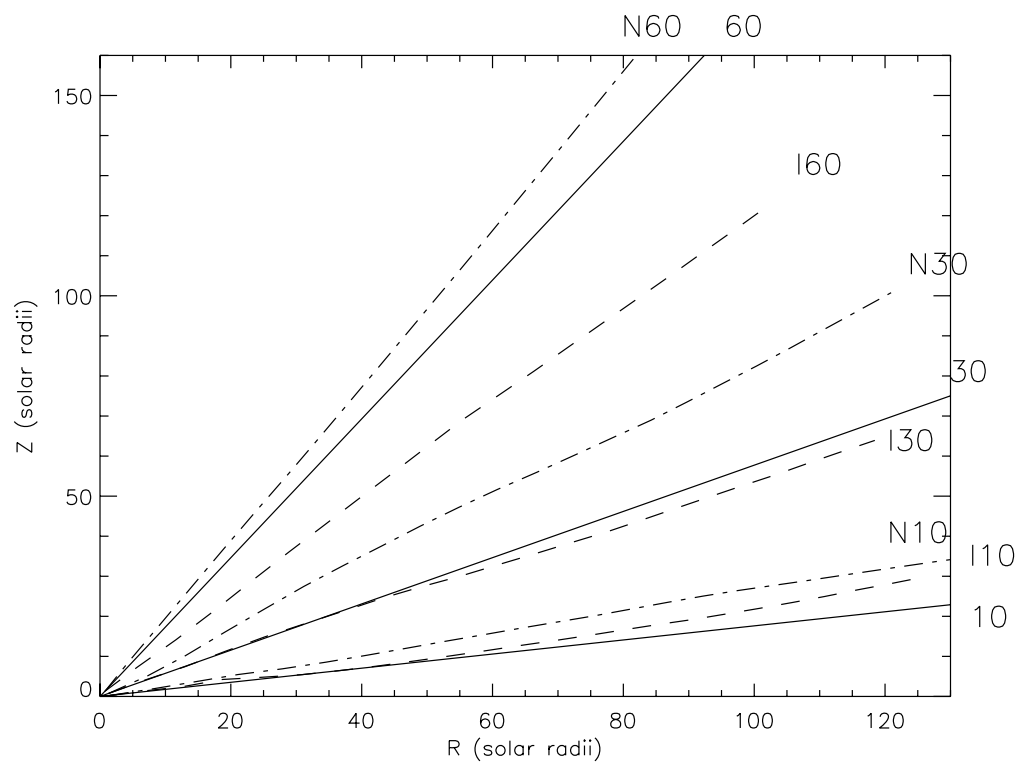

Figure 9. Evolution path of the center of relative mass for inverse (dashed lines) and normal (dasheddot lines) CMEs. The CMEs were launched on $10^{\circ}$ (in blue), $30^{\circ}$ (in green), and $60^{\circ}$ (in red). The solid lines show the initial launch angles. 

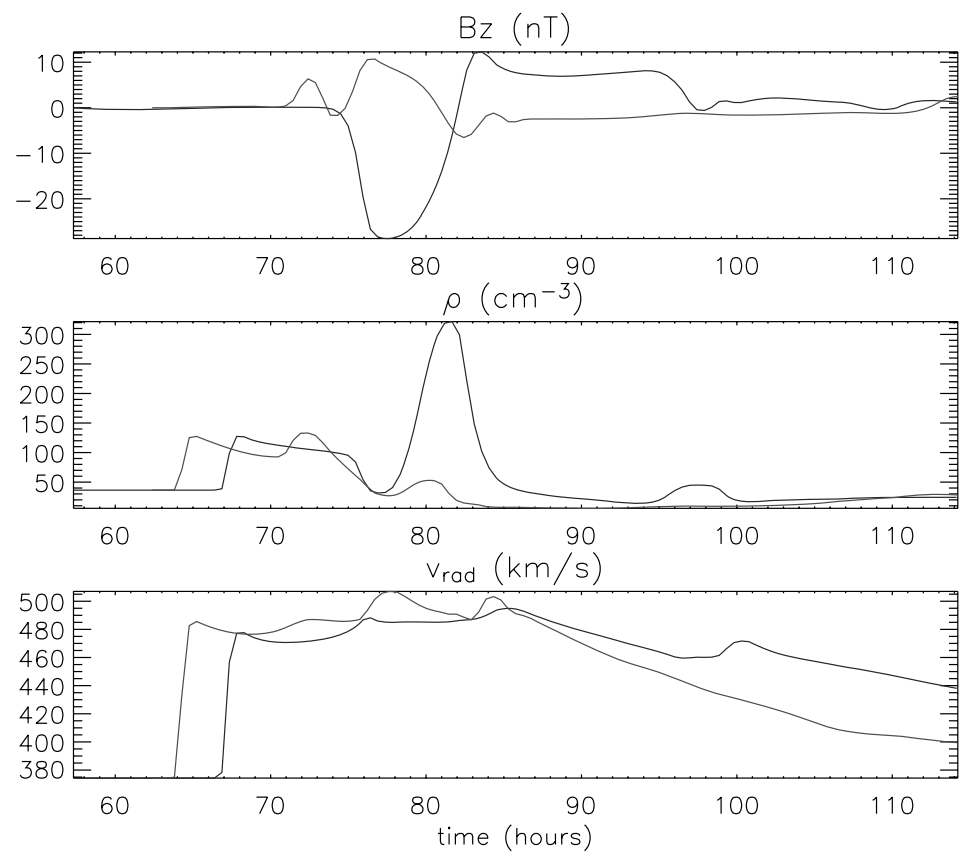

Figure 10. Evolution of the magnetic field, density and velocity in time in the equatorial plane $\left(\theta=0^{\circ}\right)$ at a position of $215 R_{\odot}$ for a $\mathrm{CME}$ launched $\theta_{\mathrm{cme}}=10^{\circ}$ above the equator; red lines: normal CME; blue lines: inverse CME.

inverse and normal CMEs launched at $10^{\circ}, 30^{\circ}$, and $60^{\circ}$. For the CMEs at $30^{\circ}$ and $60^{\circ}$, the normal CMEs are deviated towards the pole while the inverse CMEs are deviated towards the equatorial plane. Surprisingly the inverse CME launched at $10^{\circ}$ is deviated away from the equatorial plane. This is caused by the density repartition in the wind (see Figure 2) and was remarked earlier by Smith et al. (1997). The higher density close to the equator acts like a natural shield and protects the Earth from the incoming CMEs. However, the density of this wind model is too high in the equatorial plane (see later) and this probably amplifies this phenomenon. Nevertheless, in our simulations, an inverse CME is always travelling closer to the equatorial plane than a normal one and is thus more dangerous for the Earth.

Figure 10 presents data, from our numerical simulations, that would have been recorded by a satellite at $1 \mathrm{AU}$ for CMEs launched at $10^{\circ}$. It is obvious from this figure that the magnetic cloud (an increase of the magnetic field strength followed by an increase of the density) misses the Earth in the case of a normal CME and hits the Earth for the inverse CME. This is a clear example showing that we need information on the initial polarity of a CME to predict its geo-effectiveness. 


\section{Conclusions}

In this paper, we first presented three solar wind models. We discussed CME initiation models based on magnetic foot point shearing and magnetic flux emergence. In spite of extensive parameter studies, however, these models were not able to produce the fast CMEs that create shock waves in the IP space and are, therefore, important for space weather. For the CME evolution studies we thus applied the much simpler, so-called 'density-driven', CME model. We then extended the study of Chané et al. (2005) on the influence of the initial magnetic polarity on the evolution of CMEs into the IP space by extending the computational domain up to 1 AU. We have shown that the evolution path of the CMEs was strongly related to the initial magnetic polarity. An example was given where an inverse CME hits the Earth and where the same CME but with a normal magnetic polarity misses the Earth. We have also shown that the time of arrival was influenced by the initial magnetic polarity: A normal CME propagates faster than an inverse CME and thus reached the Earth a few hours earlier. Last but not the least, the inverse CMEs display a strong southward magnetic field (a well known source of magnetic storms) at $1 \mathrm{AU}$. The geo-effectiveness of a CME is thus strongly related to its magnetic polarity.

\section{Acknowledgements}

These results were obtained in the framework of the projects GOA 2004/01 and OT 02/57 (K. U. Leuven), G.0451.05 (FWO-Vlaanderen) and 90203 (ESA Prodex 8).

\section{References}

Amari, T., Luciani, J. F., Aly, J. J., Mikic, Z., and Linker, J.: 2005, Astrophys. J. 595, 1231-1250.

Chané, E., Jacobs, C., van der Holst, B., Poedts, S., and Kimpe, D.: 2005, Astrom. Astrophys. 432, 331.

Chané, E., van der Holst, B., and Poedts, S.: 2005, Astrom. Astrophys., in press.

Chen, P. F. and Shibata, K.: 2000, Astrophys. J. 545, 524.

Dubey, G., Poedts, S., and Van der Holst, B.: 2005, Procedings of the Solar Wind11/Soho 16, Whistler, Canada, 11-17 June, 2005, ESA SP-592, in press.

Gombosi, T. I., DeZeeuw, D. L., Groth, C. P. T., Powell, K. G., and Stout, Q. F.: 2000, J. of Atmos. Terrest. Phys. 62, 1515.

Gopalswamy, N., Barbieri, L., Lu, G., Plunkett, S. P., and Skoug, R. M.: 2005, Geophys. Res. Lett. 32, 3 .

Groth, C. P. T., De Zeeuw, D. L., Gombosi, T. I., and Powell, K. G.: 2000, JGR 105, 25053-25078.

Jacobs, C., Poedts, S., Van der Holst, B., and Chané, E.: 2005, Astrom. Astrophys. 430, 1099.

Jacobs, C., Poedts, S., and Van der Holst, B.: 2005, Proc. Solar Wind11/Soho 16, Whistler, Canada, 11-17 June, 2005, ESA SP-592, in press. 
Jacobs, C., Poedts, S., and Van der Holst, B.: 2006, CME Triggering by magnetic foot point shearing, Astrom. Astrophys. in press.

Keppens R. and Goedbloed, J. P.: 1999, Astrom. Astrophys. 343, 251.

Klimchuk, J. A.: 2001, Procedings of the Chapman Conference on Space Weather, AGU, Geophysical Monograph Series 125, eds. Song, P., Singer, H., and Siscoe, G., 143-157.

Lee, C. O., Ledvina, S. A., Luhmann, J. G., Odstrcil, D., Riley, P., Crary, F., et al.: 2004, AGU Fall Meet. Abstr., A1418.

Lionello, R., Linker, J. A., and Mikić, Z.: 2003, AIP Conf. Proc. 679: Solar Wind Ten, 222-225.

Lionello, R., Riley, P., Linker, J. A., and Mikić, Z.: 2005, Astrophys. J. 625, 463L.

Lugaz, N., Manchester W. B., and Gombosi T. I.: 2005, Astrophys. J. 627, 1019-1030.

Low, B. C. and Zhang, M.: 2002, Astrophys. J. 564, L53.

Manchester, W. B., Gombosi, T. I., Roussev, I., et al.: 2004a, J. Geophys. Res. (Space Phys.) 109, 1102.

Manchester, W. B., Gombosi, T. I., Roussev, I., et al.: 2004b, J. Geophys. Res. (Space Phys.) 109, 2107.

Mikić Z. and Linker, J. A.: 1994, Astro Phy. J. 430, 898.

Odstrcil, D., Riley, P., and Zhao, X. P: 2004, JGR 109, A18.

Odstrcil, D., Pizzo, V. J., and Arge, C. N.: 2005, JGR 109, A18.

Riley, P., Linker, J. A., Mikić, Z., et al.: 2002, Astrom. Astrophys. 578, 972.

Roussev, I. I., Gombosi, T. I., Sokolov, I. V., Velli, M., Manchester, W. B., De Zeeuw, D. L., et al.: 2003a, Astrophys. J. 595, L57-L61.

Smith, Z., Watari, S., Dryer, M., Manoharan, P. K., Mclntosh, P. S.: 1997, SPh 171, 177-190.

Tóth, G.: 1996, Astrophys. Lett. Comm. 34, 245.

Tsurutani, B., Wu, S. T., and Zhang, T. X., Dryer, M.: 2003, Astrom. Astrophys. 412, 293.

Vanselow, K. H. and Ricklefs, K.: 2005, J. Sea Res. 53, 319.

Wu, C.-C. and Lepping, R. P.: 2005, J. Atmos. Terrest. Phys. 67, 283. 